\title{
Síndrome neuroléptico maligno-like como complicación en enfermedad de Parkinson. Desenlace fatal. Reporte de un caso y revisión de la literatura
} Case report of an 80 year-old patient with Parkinson's Disease affected by Malignant Hyperthermia Syndrome with fatal outcome

Carlos Salinas S. ${ }^{1}$, Amalia Laso F. ${ }^{2}$ y Alberto Vargas C. ${ }^{3}$

\begin{abstract}
Parkinson's disease presents its particular complications and others associated with pharmacological therapy. In this report we present an 80 year-old patient with Parkinson's disease, who having a febrile syndrome decides to suspend his usual antiparkinsonian treatment. As a consequence an exacerbation of their extrapyramidal symptoms appear and his fever syndrome persists without infectious focus. Neuroleptic Malignant Syndrome variant with fatal outcome was concluded.
\end{abstract}

Key words: Parkinson's disease, malignant hyperthermia, levodopa.

Rev Chil Neuro-Psiquiat 2014; 52 (2): 89-92

\section{Introducción}

Se ha descrito un cuadro similar al Síndrome SNeuroléptico Maligno (SNM) en pacientes con Enfermedad de Parkinson (EP), principalmente asociado a la descontinuación abrupta o la reducción de la dosis habitual durante el tratamiento con levodopa ${ }^{1}$. Esto se ha denominado Síndrome
Neuroléptico Maligno-like (SNM-L) ${ }^{2,3}$. Se describió por vez primera en 1981, caracterizándose por hipertermia, rigidez muscular e inestabilidad autonómica que se desarrolla entre $18 \mathrm{~h}$ y 7 días después de suspendida la droga, con una mortalidad de hasta el $4 \%{ }^{4}$. Entre los datos de laboratorio que ayudan al diagnóstico, está la CK Total elevada, leucocitosis y mioglobinuria ${ }^{5}$.

Recibido: 27/03/2014

Aceptado: 13/06/2014

Los autores no presentan ningún tipo de conflicto de interés.

1 Médico, Servicio de Medicina Interna, Hospital Luis Tisné Brousse.

2 Médico, Servicio de Medicina Interna, Hospital Luis Tisné Brousse.

3 Neurólogo, Servicio de Medicina Interna, Hospital Luis Tisné Brousse 
En la literatura médica, se le ha dado múltiples denominaciones: Hipertermia debida a suspensión de levodopa, Síndrome Parkinsonismo-Hipertermia, Síndrome dopaminérgico maligno o Síndrome de depleción aguda de dopamina.

La fisiopatología del SNM no está del todo clara aún. Se cree que una disminución aguda de levodopa en el hipotálamo, sistema nigroestatrial y en las vías dopaminérgicas mesocorticales son los causantes del desarrollo de este síndrome.

Se ha otorgado un rol al ácido homovalínico (AHV), metabolito terminal de la levodopa, que podría tener relación con los que se cree son un subtipo de pacientes que desarrollan SNM-L. Se ha visto que estos pacientes tendrían menores concentraciones que lo esperable de AHV en el líquido cefaloraquídeo al suspender la droga antiparkinsoniana $^{6,7}$.

Se han clasificado los síndromes que producen hipertermia por drogas, en los siguientes: Síndrome Serotoninérgico, Síndrome Simpaticomimético, Síndrome Neuroléptico Maligno, Síndrome Anticolinérgico, y la Hipertermia Maligna, estos dos últimos relacionados con drogas usadas en parkinsonismos, como la memantina y anticolinérgicos ${ }^{8}$. En general, estas entidades clínicas responden a una respuesta hipermetabólica caracterizada por aumento extremo de la temperatura, rigidez muscular, sudoración, taquicardia y compromiso de conciencia como resultado de la exposición a una droga determinada en individuos susceptibles, pero, a diferencia del SNM-L, éste ocurre a la primera exposición del fármaco; generalmente anestésicos.

\section{Caso clínico}

Paciente sexo masculino de 80 años, portador de Enfermedad de Parkinson de 10 años de evolución, en tratamiento actual con levodopa/carbidopa 250/25 mgs en esquema 3/4-3/4-1-al día; además, secuelado con hemiparesia faciobraquiocrural izquierda secundaria a accidente vascular encefálico tipo lacunar; igualmente, con hipotiroidismo en tratamiento suplementario con Levotiroxina 100 mcgs/día; y Depresión tratada con Citalopram 20 mgs/día.

Paciente consulta por presentar compromiso de estado general progresivo, fiebre cuantificada hasta $38^{\circ} \mathrm{C}$, y diarrea circunscrita a un día. Evaluado por atención domiciliaria de urgencias, se deriva a nuestro hospital por deshidratación importante.

Ingresa al Servicio de Urgencias hipertenso, eucárdico, febril de $38^{\circ} \mathrm{C}$, eupnéico, deshidratado, comprometido cuali-cuantitativamente de conciencia. Sin focalidad neurológica de vías largas.

Del laboratorio de ingreso destacan gases sanguíneos arteriales normales, electrolitos plasmáticos normales, función renal normal, perfil hepático normal, lactato $12 \mathrm{mg} / \mathrm{dL}$, leucocitos séricos 15.200 con $85 \%$ de segmentados, PCR 1,9 $(\mathrm{VN}<1)$, VHS $20 \mathrm{~mm} / \mathrm{h}$, glucosa sérica normal, pruebas de coagulación normales, LDH $809 \mathrm{U} / \mathrm{L}$, CK total elevada en rango de 510 U/L (VN 55-170), hormonas tiroideas normales. Sedimento urinario sin alteraciones.

Se sospecha fiebre de origen infeccioso de posible foco abdominal y se inician antibióticos endovenosos empíricos e hidratación.

Posteriormente se completa estudio: urocultivo negativo, hemocultivos seriados negativos, radiografía de tórax normal. RPR no reactivo. Antígeno prostático específico normal. Se realiza punción lumbar que resulta no sugerente de infección: leucocitos 0 , diferencial no se realiza por bajo recuento, proteínas $40 \mathrm{mg} / \mathrm{dL}$, glucosa $49 \mathrm{mg} / \mathrm{dL}$. Gram sin bacterias.

Se toma TAC de Abdomen y Pelvis con contraste, donde no se evidencian procesos infecciosos $u$ otras anormalidades.

Evoluciona en malas condiciones generales, manteniendo compromiso de conciencia, hemodinámicamente inestable; taquicárdico, hipertenso, oligúrico, febril persistente, requiriendo oxígeno suplementario.

Se rescata antecedente de suspensión de medicamento antiparkinsoniano 2 días previos a la evaluación en domicilio y 2 días posteriores a su ingreso hospitalario. En ese momento, se reinicia Grifoparkin en dosis habitual y se suspende Citalopram. 
Paciente persiste febril hasta $40,5^{\circ}$, con rigidez en rueda dentada generalizada y miocloníasmultifocales al examen físico. Se sospecha SNM-L y se inicia Bromocriptina 5,0 mgs c/6 h.

Se indican medidas de neuroprotección y manejo agresivo de fiebre. Por epidemiología, se decide tratamiento empírico para Meningitis Bacteriana Aguda hasta obtener cultivos y PCR virales, que finalmente resultaron negativos. Se agrega cobertura antiviral en dosis meníngeas.

El paciente empeora clínicamente en el transcurso de las horas siguientes; taquicárdico, con requerimientos de hasta $100 \%$ de oxígeno suplementario, completando $72 \mathrm{~h}$ de fiebre persistente.

Finalmente, presenta paro cardiorrespiratorio, reportándose fallecimiento al día 3 de su ingreso.

\section{Comentario}

En el SNM-L el diagnóstico precoz es fundamental. Una vez diagnosticado, debe iniciarse un manejo de soporte activo; hidratación, disminución agresiva de la temperatura, relajantes musculares y monitorización continua, asociada a una reinstalación inmediata del fármaco dopaminérgico a dosis iguales a la habitual utilizada por el paciente ${ }^{9}$ con el fin de yugular la sintomatología. Se puede agregar bromocriptina (7,5 a 15 mgs/día), y dantroleno para el relajo muscular con una dosis inicial de $2 \mathrm{mg} / \mathrm{kg}$ ev e indicación de repetir hasta que los síntomas cedan ${ }^{10}$, pero no hay estudios que avalen la eficacia de ambos ${ }^{11}$.

En nuestro caso, se manejaron varios diagnósticos diferenciales antes de plantear el definitivo, por lo que al momento de la instalación de la terapia más correcta el cuadro estaba ya muy avanzado.

Complicaciones causantes de mortalidad y mal pronóstico en estos pacientes son rabdomiolisis y falla renal aguda concomitante, falla respiratoria aguda secundaria a tromboembolismo pulmonar o a neumonía aspirativa, convulsiones, encefalopatía hipóxica isquémica, infarto al miocardio, coagulación intravascular diseminada, falla hepática fulminante y sepsis ${ }^{11}$.

Hay similitud de síntomas con el Síndrome Serotoninérgico desde el punto de vista cognitivo, autonómico y neuromuscular; pero en nuestro caso se eliminó esta variable al suspender el ISRS a su ingreso.

En conclusión, debe considerarse un SNM-L en todo paciente afectado con EP que presente un rápido deterioro de sus síntomas, con rigidez e hipertermia en relación al antecedente de una modificación o suspensión reciente de su tratamiento dopaminérgico habitual.

\section{Resumen}

La Enfermedad de Parkinson presenta complicaciones propias de su patología y asociadas a su terapia farmacológica. Presentamos un paciente de 80 años con Enfermedad de Parkinson, que debuta con un sindrome febril por lo que suspende su tratamiento antiparkinsoniano habitual. Como consecuencia, se agrega exacerbación de su clínica extrapiramidal, persistiendo su cuadro febril sin foco infeccioso. Se concluye variante del Síndrome Neuroléptico Maligno con resolución fatal.

Palabras clave: Enfermedad de Parkinson, hipertemia maligna, levodopa. 


\section{Referencias bibliográficas}

1. Sato Y, Honda Y, Asoh T, Iwamoto J, Kanoko T, Satoh K. Cardiac involvement in malignant syndrome in Parkinson's disease. Eur Neurol 2005; 54: 88-92.

2. Toru M, Matsuda O, Makiguchi K, Sugano K. Neuroleptic malignant syndrome-like state following a withdrawal of antiparkinsonian drugs. J Nerv Ment Dis 1981; 169: 324-7.

3. Friedman JH, Feinberg SS, Feldman RG.A neuroleptic malignantlike syndrome due to levodopa therapy withdrawal. JAMA 1985; 254: 2792-5.

4. Factor SA, Santiago A. Parkinsonism-hyperpyrexia syndrome in Parkinson's disease. In: Movement disorder emergencies: Diagnosis and treatment. New York: Humana Press; 2005. p. 29-41.

5. Carbone J. The neuroleptic malignant and serotonin syndromes. Emerg Med Clin North Am 2000; 18: 317-25.
6. Ueda M, Hamamoto M, Nagayama H, Otsubo K, Nito C, Miyazaki T, et al. Susceptibilitytoneurolepticmalignantsyndrome in Parkinson'sdisease. Neurology 1999; 52: 777-81.

7. Biochemical alterations during medication withdrawal in Parkinson's disease with and without neuroleptic malignant-like syndrome. J NeurolNeurosurg Psychiatry 2001; 71 (1): 111-3.

8. Diagnosis and treatment of drug-induced hyperthermia. Am J Health Syst Pharm 2013; 70 (1): 34-42.

9. The Parkinsonism-Hyperpyrexia Syndrome. Neurocrit Care 2009; 10: 136-40.

10. Chandran G, Mikler J, Keegan D. Neuroleptic malignant syndrome: case report and discussion. CMAJ 2003; 169: 439-42.

11. Ikebe S, Harada T, Hashimoto T, et al. Prevention and treatment of malignant syndrome in Parkinson's disease: a consensus statement of the malignant syndrome research group. Parkinsonism. Relat Disord 2003; 9 Suppl 1: S47-9.

Correspondencia:

Carlos Salinas S.

Servicio de Medicina Hospital Luis Tisné B., Santiago, Chile.

Teléfono: 4725331

E-mail: csalinass@gmail.com 